\title{
(6) OPEN ACCESS \\ Effect of body position and ventilation on umbilical artery and venous blood flows during delayed umbilical cord clamping in preterm lambs
}

\author{
Stuart B Hooper, ${ }^{1,2}$ Kelly J Crossley, ${ }^{1,2}$ Valerie A Zahra, ${ }^{1}$ Jeroen van Vonderen, ${ }^{3}$ \\ Alison Moxham, ${ }^{1}$ Andrew W Gill, ${ }^{4}$ Martin Kluckow, ${ }^{5}$ Arjan B te Pas, ${ }^{3}$ \\ Euan M Wallace, ${ }^{1,2}$ Graeme R Polglase ${ }^{1,2}$
}

${ }^{1}$ Ritchie Centre, The Hudson Institute for Medical Research, Clayton, Victoria, Australia Department of Obstetrics and Gynaecology, Monash University, Clayton, Victoria, Australia

${ }^{3}$ Department of Pediatrics, Leiden University Medical Centre, Leiden, The Netherlands ${ }^{4}$ Centre for Neonatal Research and Education, The University of Western Australia, Crawley, Western Australia, Australia ${ }^{5}$ Department of Neonatology, Royal North Shore Hospital and University of Sydney, Sydney, New South Wales, Australia

\section{Correspondence to}

Professor Stuart B Hooper, The Ritchie Centre, Hudson Institute of Medical Research, 27-31 Wright Street, Clayton, VIC 3168, Australia;

stuart.hooper@monash.edu

Received 9 May 2016 Accepted 13 October 2016

Published Online First

8 November 2016

\section{ABSTRACT \\ Objective While delayed umbilical cord clamping} (UCC) is thought to facilitate placental to infant blood transfusion, the physiological factors regulating flow in the umbilical arteries and veins during delayed UCC is unknown. We investigated the effects of gravity, by changing fetal height relative to the placenta, and ventilation on umbilical blood flows and the cardiovascular transition during delayed UCC at birth. Methods Catheters and flow probes were implanted into preterm lambs (128 days) prior to delivery to measure pulmonary, carotid, umbilical artery (UaBF) and umbilical venous (UvBF) blood flows. Lambs were placed either $10 \mathrm{~cm}$ below or $10 \mathrm{~cm}$ above the ewe. Ventilation commenced 2-3 min before UCC and continued for 30 min after UCC.

Results Gravity reduced umbilical and cerebral flows when lambs were placed below the midline, but the reduction in UaBF and UvBF was similar. Ventilation during delayed UCC reduced UvBF and UaBF by similar amounts, irrespective of the lamb's position, such that flows into and out of the placenta remained balanced. The effects of ventilation on umbilical flows were much greater than the effects of gravity, but no net placental to lamb blood transfusion could be detected under any condition. Cardiovascular parameters, cerebral oxygen kinetics and final blood volumes were similar in both groups 5 min after UCC.

Conclusions Gravity caused small transient effects on umbilical and cerebral flow, but given changes were similar in umbilical arteries and veins, no net placental transfusion was detected. Ventilation during delayed UCC has a markedly greater influence on cardiovascular function in the newborn.

\section{INTRODUCTION}

Both clinical and experimental evidence have challenged the concept that umbilical cord clamping (UCC) should occur immediately after birth. As a result, WHO and the International Liaison Committee on Resuscitation now recommend that UCC should be delayed for at least a minute after birth in healthy term infants. ${ }^{1}$ These recommendations are largely based on the assumption that 'delayed UCC' facilitates a time-dependent transfer of blood from the placenta to baby, resulting in an increase of $\sim 8 \mathrm{~mL} / \mathrm{kg} / \mathrm{min}^{2}$ However, recent studies in animals ${ }^{3} 4$ and humans ${ }^{5}$ question the physiological circumstances under which a net transfer of blood will occur between the placenta

\section{What is already known on this topic?}

Placing an infant above or at the same height as the placenta during delayed cord clamping does not alter placental to fetal blood transfusion.

- Initiating ventilation prior to umbilical cord clamping stabilises the cardiovascular transition at preterm delivery.

\section{What this study adds?}

- Changing body position above or below the placenta during preterm delivery has minimal, and only transient effects, on umbilical and cerebral blood flow.

- The initiation of ventilation has a greater influence on umbilical and cardiovascular blood pressures and flows than changing body position.

- No net change in blood volume was detected when preterm lambs were placed above or below the ewe during delayed umbilical cord clamping.

and infant during delayed UCC. These studies show that ventilation and the associated increase in pulmonary blood flow (PBF) are key determinants of cardiac output and influence blood flow in both the umbilical arteries and veins after birth. ${ }^{5}$ However, the effect of other factors such as gravity are unknown, although a recent clinical trial has found no difference in blood transfer during delayed UCC when the infant is placed at the same height or above the introitus. ${ }^{6}$

In the fetus, PBF is low and so venous return (preload) to the left ventricle (LV) is mostly derived from umbilical venous flow via the ductus venosus and foramen ovale. ${ }^{7}$ Indeed, the placental circulation receives $30 \%-50 \%$ of fetal cardiac output and represents a low resistance pathway for fetal systemic blood flow. As such, UCC before ventilation onset not only reduces venous return (by 30\%$50 \%$ ), it also greatly increases peripheral resistance, resulting in an increase in arterial pressure. ${ }^{8} 9$ Thus, following UCC, the combined effect of a 
reduction in preload and an increase in afterload, greatly reduces cardiac output, which remains low until the lungs aerate and PBF increases. ${ }^{3}{ }^{10}$ Aerating the lung and increasing PBF before UCC allows PBF to immediately replace umbilical venous return as the primary source of LV preload following UCC and thereby avoids the large swings in cardiac output associated with UCC.

Although the benefits of delayed UCC are mainly attributed to net placental to infant blood transfusion, little information is available on the factors regulating umbilical blood flows into and out of the infant during UCC. A recent study in human infants has shown that, during UCC, umbilical venous flow is intermittent, markedly increasing during deep inspiratory efforts and ceasing or reversing during crying, ${ }^{5}$ presumably due to pressure changes within the abdomen and thorax. Umbilical arterial flow was also reduced and sometimes ceased during crying and at times flow became bi-directional, resulting in flow towards the placenta during systole and towards the infant during diastole. ${ }^{5}$ Although that study highlighted the influence of ventilation on umbilical arterial and venous blood flows, all infants were placed above the introitus, on the mother's abdomen. As such, how gravity may influence the effect of ventilation on these blood flows is unknown.

It is commonly assumed that placing the infant below the introitus during delayed UCC increases umbilical venous flow into the infant and reduces umbilical arterial flow into the placenta. This is consistent with the report of increased blood volumes in infants placed below the introitus during delayed UCC. $^{2}$ However, this ignores the concept that arterial and venous vessels constitute a circuit, whereby flows into and out of the placenta and infant should balance, resulting in no continuing blood transfer. In this study, we have investigated the effect of ventilation and gravity (by altering the vertical height of the newborn relative to the placenta) on blood flow in the umbilical arteries and veins during delayed UCC. We hypothesised that ventilation onset will reduce flow in both vessels and that gravity will also affect flow in both vessels, but that the change will be similar, resulting in little or no net movement of blood between the infant and placenta.

\section{MATERIALS AND METHODS}

All experimental procedures on animals were approved by Monash University's Monash Medical Centre (A) Animal Ethics Committee.

\section{Surgical preparation}

Aseptic surgery was performed on Pregnant Border-Leicester ewes at $128 \pm 1$ days gestation (term is $\sim 147$ days) to implant flow probes and catheters and to deliver the lamb as described previously. ${ }^{10}$ Anaesthesia of the ewe and fetus was induced with an intravenous bolus of 5\% sodium thiopentone (Pentothal; $1 \mathrm{~g}$ in $20 \mathrm{~mL}$ ) and, following intubation, maintained with inhalation of $1.5 \%-3 \%$ isoflurane in a blended oxygen/air mixture. The fetal head and neck were exposed via a hysterotomy and an ultrasonic flow probe $(3 \mathrm{~mm}$; Transonic Systems, Ithaca, New York, USA) was placed around a carotid artery. Polyvinyl catheters were inserted into the other carotid artery and into a jugular vein. Ultrasonic flow probes $(4 \mathrm{~mm})$ were placed around an umbilical artery and vein and the left pulmonary artery, which was accessed via a left thoracotomy. ${ }^{11}{ }^{12}$ The lamb's trachea was intubated with a $4.0 \mathrm{~mm}$ cuffed endotracheal tube and a near-infrared spectroscopy (NIRS) probe was placed on the lamb's head overlying the left frontal cortex. ${ }^{4}$ A pulse-oximeter sensor was placed on the lamb's right forelimb to measure preductal transcutaneous oxygen saturation $\left(\mathrm{SpO}_{2}\right)$ levels and arterial pressures were measured using pressure transducers (PD10; DTX Plus Transducer; Becton Dickinson, Singapore). All physiological data were recorded digitally using a data acquisition system (Powerlab; ADInstruments, Castle Hill, Australia).

\section{Experimental procedure}

A known number of biotin-labelled red blood cells (RBCs) were injected into the lamb $\sim 10 \mathrm{~min}$ before a venous blood sample was collected to measure the combined blood volume of the lamb and its placenta. The lamb was then fully delivered and placed at the same vertical height as the mid-abdomen level of the ewe, taking care to not disrupt blood flow in the umbilical vessels. Following a brief stabilisation period, the lamb was placed at either (i) $10 \mathrm{~cm}$ above the ewe's mid abdomen level ( $\mathrm{n}=8$; above) or (ii) $10 \mathrm{~cm}$ below the ewe's mid abdomen level $(n=6$; below). Allocation of lambs into each group was randomised. Following repositioning, all physiological recordings were allowed to stabilise before ventilation commenced. As the umbilical cord is shorter in sheep than in humans, the differences in vertical height above and below the ewe were restricted to $\pm 10 \mathrm{~cm}$ to ensure that the cord was not placed under tension and flow in the umbilical vessels was not compromised.

In all lambs, pulmonary ventilation commenced while the umbilical cord remained intact and patent so that the effect of ventilation and the position of the lamb on blood flow in the umbilical vessels could be assessed. UCC was delayed until after PBF had increased ( 2-3 min) whereupon the cord was clamped and cut. In all lambs, ventilation began with a 20 s sustained inflation delivered by a Neopuff (Fisher \& Paykel Healthcare, Panmure, Auckland, New Zealand) using a peak inflation pressure of $35 \mathrm{cmH}_{2} \mathrm{O}$. Lambs then received positive pressure ventilation (Babylog 8000+, Dräger, Lübeck, Germany) in volume guarantee mode with a tidal volume of $7 \mathrm{~mL} / \mathrm{kg}$ and a positive end-expiratory pressure of $5 \mathrm{cmH}_{2} \mathrm{O}$. Inspired gases were warmed and humidified, the inspired oxygen content was adjusted to maintain transcutaneous arterial saturation $\left(\mathrm{SpO}_{2}\right)$ at $90 \%-95 \%$ and a $\mathrm{PaCO}_{2}$ of $35-55 \mathrm{~mm} \mathrm{Hg}$ was targeted. The lamb's well-being was monitored by regular blood gas analysis (ABL30, Radiometer, Copenhagen, Denmark) and $\mathrm{SpO}_{2}$ monitoring. All lambs were lightly anaesthetised and apneic throughout the experiments. No surfactant was given as we were primarily concerned with the cardiovascular changes at birth rather than the effects of subsequent ventilation.

Following UCC, a known volume of venous blood was collected before a known number of biotin-labelled RBCs were again injected into the lamb. After another 5-10 min mixing period, a known volume of venous blood was collected to measure the blood volume of the lamb following its separation from the placenta.

\section{Data and statistical analysis}

All pressure and flow data were averaged over $10 \mathrm{~s}$ and analysed at selected time intervals before and after the vertical height of the lamb was altered, 1 and 2 min after ventilation onset and 5 min after UCC. The combined blood volume of the lamb and placenta before UCC and blood volume of the lamb following UCC was measured in each group using the biotin-labelled RBC technique $^{13} 14$ and compared using a one-way analysis of variance (ANOVA).

$\mathrm{SpO}_{2}$ (Masimo) and $\mathrm{SctO}_{2}$ (NIRS; Casmed) were used to calculate cerebral oxygen consumption and extraction as described 
previously. ${ }^{4} 15$ Cerebral oxygen consumption $\left(\mathrm{VO}_{2}\right.$ was calculated as: mean $\mathrm{CBF} \times\left(\mathrm{SaO}_{2}-\mathrm{SctO}_{2}\right)$, where $\mathrm{CBF}$ is cerebral blood flow, measured from the carotid artery. Cerebral oxygen extraction was calculated as: $\mathrm{SaO}_{2}-\mathrm{SctO}_{2} / \mathrm{SaO}_{2}$.

All data were compared over time and between groups using either a two-way repeated measures ANOVA for postnatal physiological data, or Student's t-test for fetal data (Sigmastat V.3.0, SPSS). Data are presented as mean \pm SEM. Statistical significance was accepted for $\mathrm{p}<0.05$.

\section{RESULTS}

Fetal values prior to change in vertical height

Fetal body weights, sex distribution and arterial blood gas parameters prior to delivery were similar between groups (table 1).

Peak inspiratory pressures (figure 1A) and delivered tidal volumes were not different between groups. Alveolar arterial difference in oxygen was not different between groups at any time point (figure $1 \mathrm{~B}$ ). $\mathrm{PaCO}_{2}$ (figure $1 \mathrm{C}$ ) $\mathrm{SaO}_{2}$ and $\mathrm{PaO}_{2}$ were not different between groups at any stage of the study. Haemoglobin was not different between groups, but significantly increased at 20 and $30 \mathrm{~min}$ after delivery compared with fetal values (figure 1D).

Fetal mean umbilical venous blood flow (UvBF) and mean, peak systolic and end-diastolic umbilical artery blood flow $(\mathrm{UaBF})$ were not different between groups (figure 2). Fetal mean and end-diastolic carotid arterial blood pressure $\left(\mathrm{P}_{\mathrm{CA}}\right)$ were not different between groups. Peak systolic $\mathrm{P}_{\mathrm{CA}}$ was significantly higher in below lambs compared with above lambs prior to changing the vertical height of the lambs (figure 3). Fetal heart rate, peak systolic, end-diastolic and mean PBF (figure 3) and carotid blood flow (CaBF) (figure 4) were not different between groups.

\section{Effect of changing the lambs' vertical height on cardiovascular variables}

Placing lambs $10 \mathrm{~cm}$ above the midline did not alter umbilical, pulmonary or $\mathrm{CaBFs}$, arterial pressures or heart rate.

Placing lambs $10 \mathrm{~cm}$ below the midline reduced mean UvBF (by $16 \pm 10 \%$ ) and mean UaBF (by $\sim 21 \pm 6 \%$ ), but the percentage change between groups was not significantly different (figure $2 \mathrm{C}$ ). The fall in mean $\mathrm{UaBF}$ was predominantly driven by a decrease in end-diastolic (by $45 \pm 11 \%$; $p=0.060$ ) flow, although peak systolic (by $14 \pm 5 \%$ ) flow was also reduced. In below lambs, mean $\mathrm{CaBF}$ (figure 4A) and end-diastolic $\mathrm{CaBF}$ tended to increase by $19.2 \pm 8.9$ and $21.6 \pm 6.4 \mathrm{~mL} / \mathrm{min} / \mathrm{kg}$ $(p=0.081)$, respectively, but these did not reach statistical significance. No changes were observed in PBFs, arterial pressure or heart rate in lambs when placed $10 \mathrm{~cm}$ below the midline.

\begin{tabular}{|c|c|c|}
\hline Variables & Above placenta & Below placenta \\
\hline Number & 8 & 6 \\
\hline Males, females & $3 \mathrm{M}, 5 \mathrm{~F}$ & $4 \mathrm{M}, 2 \mathrm{~F}$ \\
\hline GA at delivery (days) & $126.6 \pm 0.2$ & $127.0 \pm 0.0$ \\
\hline Body weight (kg) & $3.4 \pm 0.2$ & $3.1 \pm 0.1$ \\
\hline $\mathrm{pH}$ & $7.18 \pm 0.04$ & $7.23 \pm 0.01$ \\
\hline $\mathrm{PaCO}_{2}(\mathrm{~mm} \mathrm{Hg})$ & $66.6 \pm 4.4$ & $61.5 \pm 2.8$ \\
\hline $\mathrm{PaO}_{2}(\mathrm{~mm} \mathrm{Hg})$ & $21.2 \pm 3.8$ & $27.8 \pm 2.7$ \\
\hline $\mathrm{SaO}_{2}(\%)$ & $50.4 \pm 10.1$ & $70.8 \pm 5.0$ \\
\hline
\end{tabular}

Effect of ventilation during delayed cord clamping on cardiovascular variables

In both groups, ventilation caused a significant reduction in mean UvBF compared with preventilation values. Mean UaBF was also significantly reduced in both groups, but the decrease tended $(\mathrm{p}=0.06)$ to be greater in below lambs ( $44 \%$ reduction) compared with above lambs $(28 \%)$ at $2 \mathrm{~min}$ after ventilation onset. The decrease in $\mathrm{UaBF}$ in below lambs was associated with a significant reduction in end-diastolic UaBF, which become predominantly retrograde (ie, reversal of flow from the placenta to the fetus) within $2 \mathrm{~min}$ of ventilation onset. Ventilation onset significantly reduced peak systolic UaBF in both above and below lambs compared with preventilation values.

Ventilation decreased mean and systolic $\mathrm{P}_{\mathrm{CA}}$, and heart rate in both above and below lambs, but these reductions were only significant in below lambs; no differences were observed between groups at each time point. Two minutes after ventilation onset, end-diastolic carotid arterial pressure was significantly reduced in both groups.

Ventilation increased mean PBF within 1 min of onset in below lambs and within $2 \mathrm{~min}$ for above lambs. The increase in mean PBF was more rapid in below lambs, resulting in significantly higher PBFs in below compared with above lambs within 2 min of ventilation onset. The increase in mean PBF coincided with a significant increase in end-diastolic PBF in all groups after ventilation onset, while peak-systolic PBF was significantly increased in below lambs only (figure 3 ).

Mean CaBF (figure 4A) was significantly reduced upon ventilation onset, with retrograde (negative) end-diastolic $\mathrm{CaBF}$ apparent in $60 \%$ of below and $50 \%$ of above lambs 2 min after ventilation onset.

\section{Effect of UCC on cardiovascular parameters}

UCC significantly increased mean, peak-systolic and enddiastolic $\mathrm{P}_{\mathrm{CA}}$ and heart rate in both groups (figure 3). Similarly, compared with postventilation values, mean, peak systolic and end-diastolic PBF was increased by cord clamping. Mean PBF tended to remain higher in below lambs compared with above lambs after cord clamping, but this difference was not significant. The number of lambs with negative end-diastolic $\mathrm{CaBF}$ was reduced (to 17\%) in the above group, but the proportion did not change in the below group.

\section{Cerebral oxygen delivery}

Cerebral oxygenation, as measured by NIRS, was not different between groups throughout the study, but increased upon ventilation onset in above lambs (figure 4B). Cerebral oxygen extraction and consumption was not different between groups at any stage of the study (figure 4C, D).

\section{Changes in blood volume before and after cord clamping}

Using a Biotin-labelled technique, we found that the blood remaining in the newborn after cord clamping was not different between groups; above: $59.9 \pm 2.6 \%$, below: $60.6 \pm 1.6 \%$ (\% of fetus+placenta). For reference, in a separate study, we have found that percentage of blood remaining in lambs following immediate UCC is $56.6 \pm 1.7$ (unpublished observations).

\section{DISCUSSION}

Based on the premise that delayed UCC increases placental to infant blood transfusion, it is commonly assumed that placing the infant below the introitus will increase placental to infant blood transfer. Recent animal studies indicate that the benefits 
A

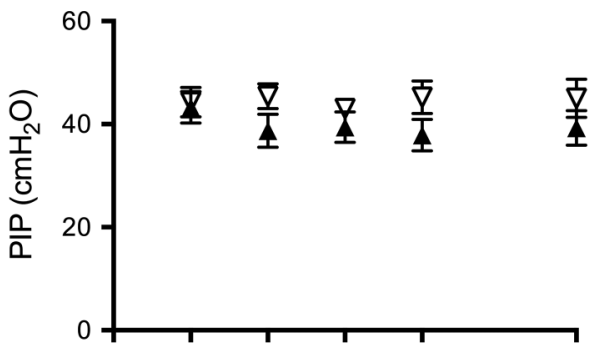

B

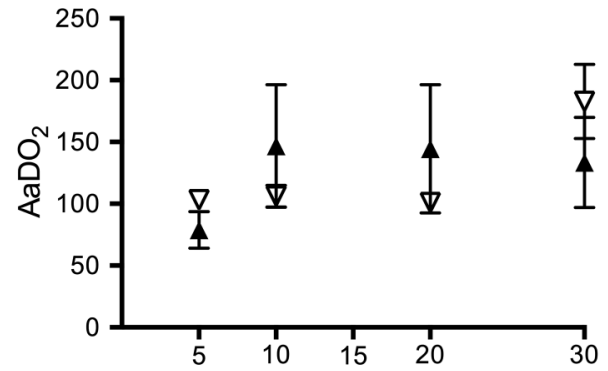

D

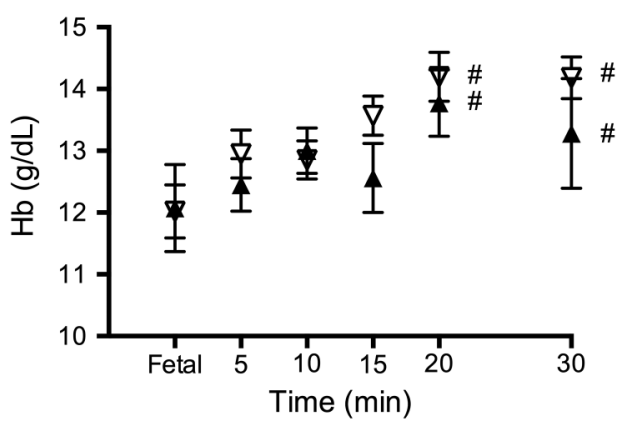

Figure 1 Ventilation parameters. (A) Peak inflation pressure (PIP), (B) alveolar-arterial difference in oxygen $\left(\mathrm{AaDO}_{2}\right),(\mathrm{C})$ partial pressure of arterial carbon dioxide $\left(\mathrm{CO}_{2}\right)$ and $(\mathrm{D})$ haemoglobin $(\mathrm{Hb})$ measured in lambs placed below (down arrows) or above (up arrows) the midline. ${ }^{\#}$ Indicates significant time effect from values before the lambs position was changed (equal).
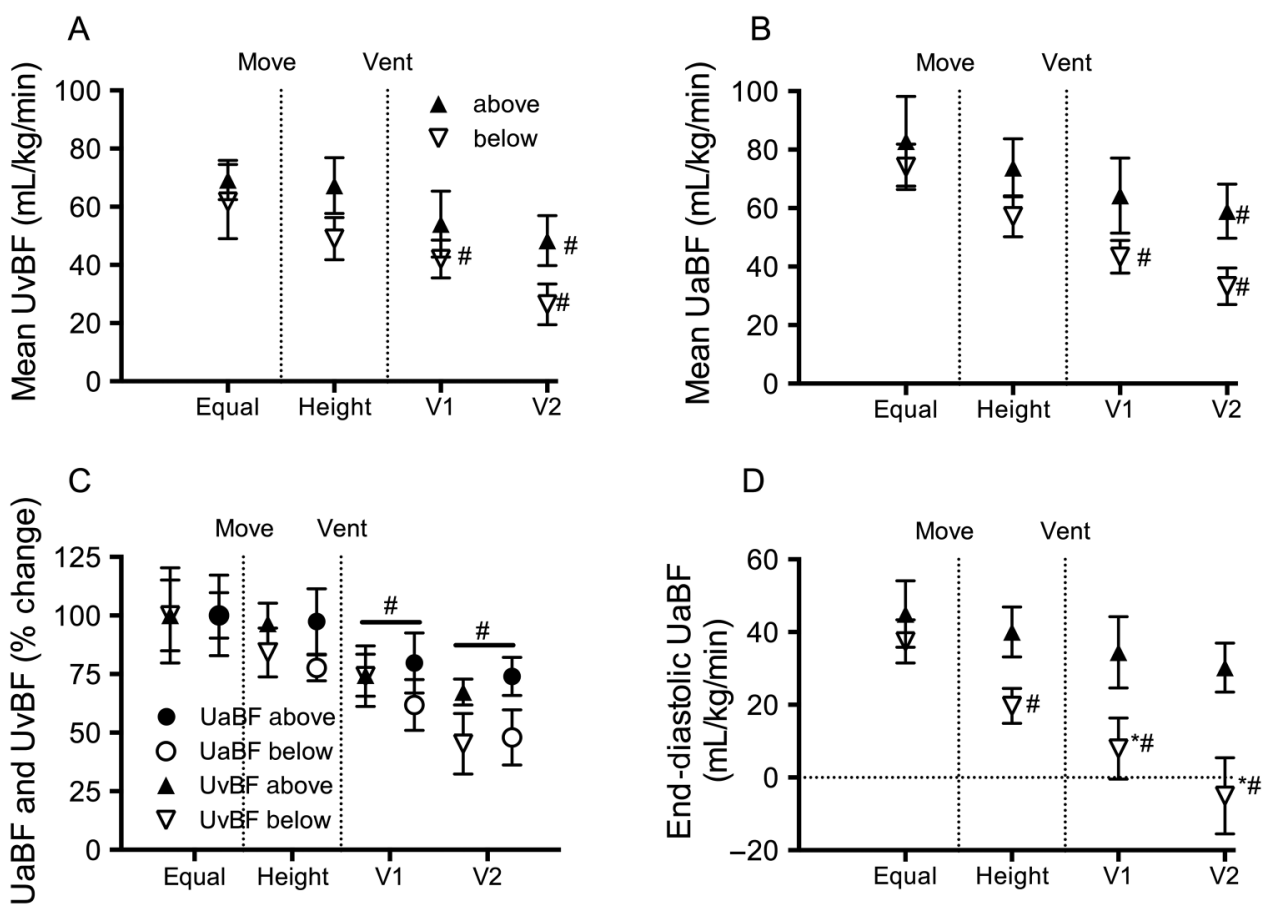

Figure 2 Umbilical blood flows. (A) Umbilical venous blood flow (UvBF), (B) mean umbilical arterial blood flow (UaBF), (C) percentage change in UvBF and UaBF and (D) end-diastolic UaBF in lambs placed below (down arrows) or above (up arrows) the midline measured prior to moving (equal), when lambs height was adjusted (height) and 1 (V1) and 2 (V2) minutes after ventilation onset. *Indicates significant differences between groups $(p<0.05)$. "Indicates significant time effect from control (equal) values.

of delayed UCC may not be due only to volume transfer. Instead, ventilation onset prior to UCC allows the supply of left ventricular preload to immediately switch from umbilical venous return to pulmonary venous return without any reduction in supply. ${ }^{3}$ However, the effect of gravity on this relationship is not known. We investigated the effect of ventilation onset and whether positioning newborn lambs above or below the mother influences UaBF and UvBF during delayed UCC. We observed only transient effects of changing body position on umbilical and cerebral flows when lambs were placed below the midline. However, the initiation of ventilation during delayed UCC had a markedly greater influence on cardiovascular 


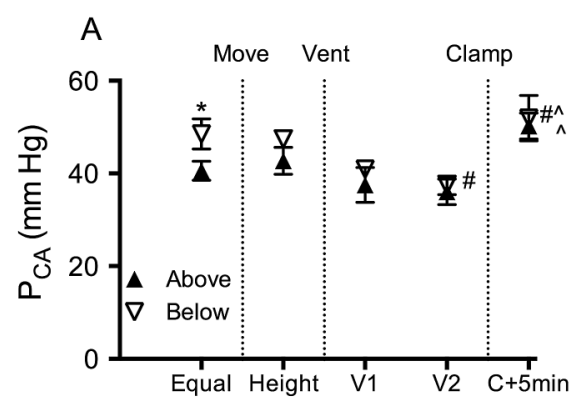

C

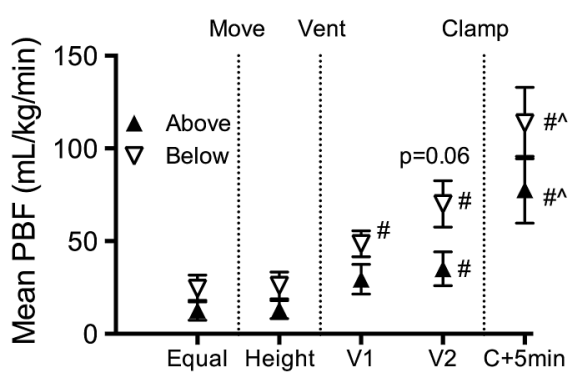

E

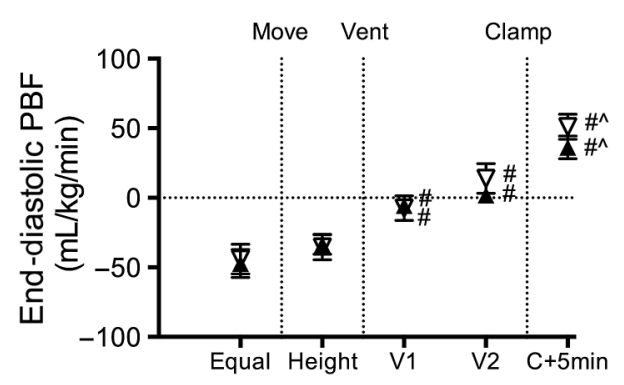

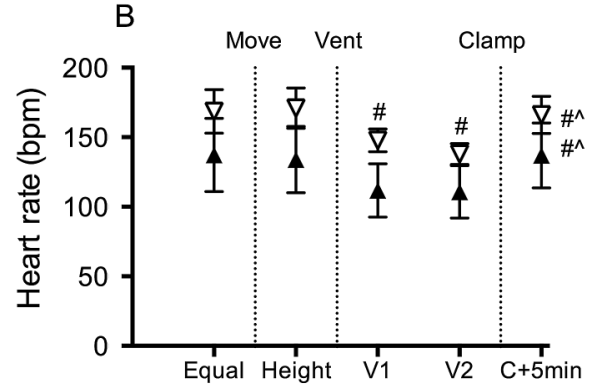

$\mathrm{D}$

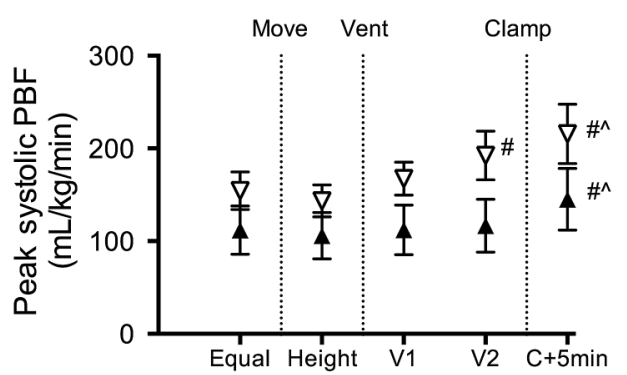

$\mathrm{F}$

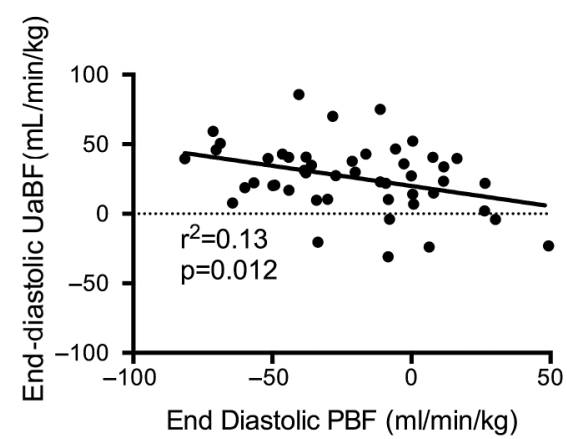

Figure 3 Systemic and pulmonary haemodynamics. $(A)$ Mean carotid arterial pressure $\left(P_{C A}\right),(B)$ heart rate, $(C)$ mean pulmonary blood flow (PBF), (D) peak systolic PBF, (E) end-diastolic PBF and (F) correlation between end-diastolic umbilical arterial blood flow (UaBF) and end-diastolic PBF in lambs placed below (down arrows) or above (up arrows) the midline measured prior to moving (equal), when lambs height was adjusted (height), 1 (V1) and 2 (V2) minutes after ventilation onset and 5 min after umbilical cord clamping ( +5 min). * Indicates significant differences between groups $(p<0.05)$. "Indicates significant time effect from control (equal) values. $\wedge$ Significant time effect between ventilation $(\mathrm{V} 2)$ and $C+5$ min.

function in the newborn. No overall effect of body position on final blood volume could be detected.

\section{Changing body position before ventilation onset}

The studies of Yao and Lind, ${ }^{2}$ indicate that during delayed UCC, gravity increases placental to infant blood transfusion, which assumes that UvBF must exceed UaBF during this time. Before ventilation onset, we found that placing the lamb below the mother reduced both $\mathrm{UaBF}$ and $\mathrm{UvBF}$ to a similar degree. Although the percentage decrease tended to be greater in UaBF than UvBF, this difference was transient and not significant as significant changes could not be detected using flow probes or by measuring changes in blood volume directly. Nevertheless, it is possible that a small transient increase in blood flow may have occurred towards the lamb when placing it below the mother. If this did occur, it was likely due to a reduction in umbilical artery input pressure combined with a syphoning pressure applied via the umbilical vein. This would reduce expansion of the placental vascular bed and shift blood volume into the lamb, although the net shift in blood must be transient.

When the lamb was placed below the mother, as UvBF decreased simultaneously with the reduction in UaBF, despite UvBF having the assistance of gravity, this reduction in UvBF can only be explained by the reduction in UaBF. This clearly demonstrates that arterial flow into the placenta is a major determinant of venous flow out of the placenta. This is consistent with the finding that placing the lamb above the mother reduced UvBF to a lesser degree than placing the lamb below the mother, despite the fact UvBF flowed against gravity when the lamb was above the mother. Similarly, the dependent interrelationship between UaBF and UvBF was further demonstrated by the finding that, when placing the lamb above the mother, UaBF tended to be reduced and not was increased when flowing with the assistance of gravity. This likely resulted from a gravityinduced reduction in UvBF, causing mild venous congestion within the placenta and an apparent increase placental vascular resistance, thereby reducing UaBF. While venous congestion may have caused a net increase in placental blood volume, we were again unable to detect this increase and so it is also likely to be small. This finding is consistent with a recent clinical trial showing that the volume of placental transfusion is not affected by placing the baby on the mother's abdomen/chest or at the same level as the introitus. ${ }^{6}$

Placing the lamb below the mother also tended to transiently increase mean and end-diastolic CaBF. Although this failed to reach statistical significance, the increase occurred in all lambs, 
A

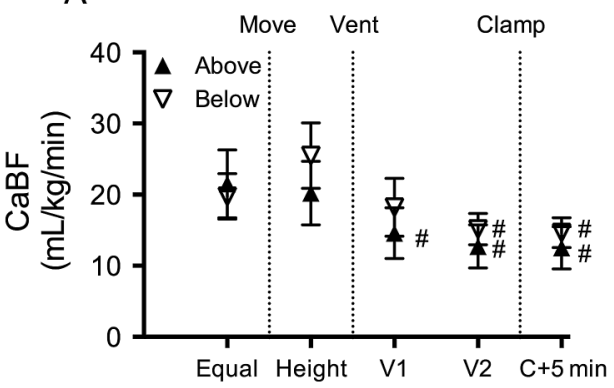

B

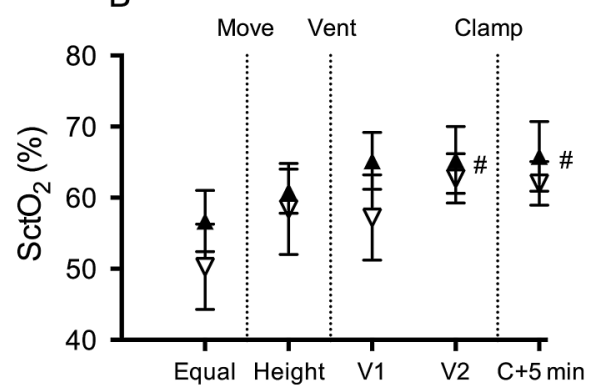

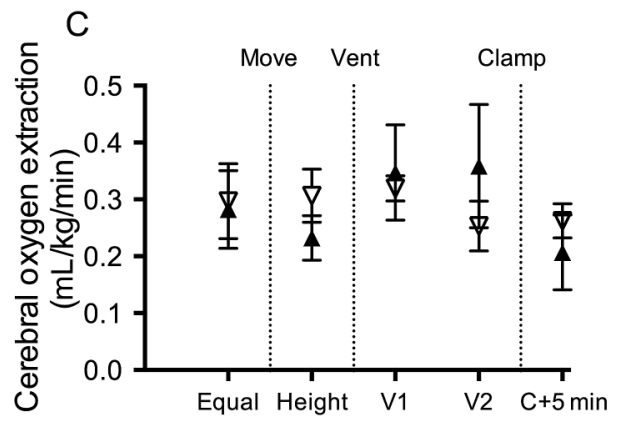

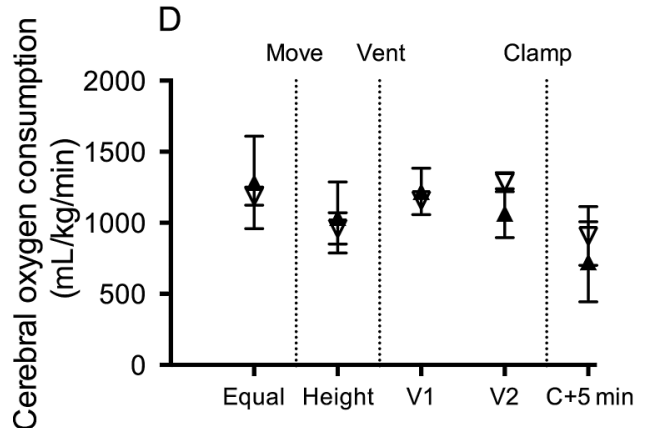

Figure 4 Cerebral haemodynamics and oxygen kinetics. (A) Carotid arterial blood flow (CaBF), (B) cerebral oxygenation as measured by near-infrared spectroscopy (NIRS) $\left(\mathrm{SctO}_{2}\right)$, (C) cerebral oxygen extraction and (D) cerebral oxygen consumption in lambs placed below (down arrows) or above (up arrows) the midline measured prior to moving (equal), when lambs height was adjusted (height), 1 (V1) and 2 (V2) minutes after ventilation onset and $5 \mathrm{~min}$ after umbilical cord clamping ( $\mathrm{C}+5 \mathrm{~min}) .{ }^{*}$ Indicates significant differences between groups $(\mathrm{p}<0.05)$. ${ }^{\#}$ Indicates significant time effect from control (equal) values. ^Significant time effect between ventilation (V2) and clamp $+5 \mathrm{~min}$.

is likely to be physiologically relevant and probably resulted from an increase in afterload caused by gravity-induced increase in umbilical artery pressure. Indeed, restricting UaBF increases arterial blood pressure and $\mathrm{CaBF}$, demonstrating the pressure passive nature of cerebral blood flow in response to rapid changes in arterial pressure. ${ }^{3}$ Our inability to detect a coincident increase in arterial blood pressure with the increase in $\mathrm{CaBF}$ most probably reflects a methodological limitation as the vertical height of the pressure transducers were changed with the height of the lamb. As a result, millimetre differences in the relative vertical heights between the lamb and the pressure transducer before and after moving the lambs likely impacted on our ability to statistically detect an increase in arterial pressure.

\section{Effects of ventilation during delayed UCC at different heights}

Initiating ventilation produced markedly greater effects on umbilical blood flows than changing the vertical height of the lamb. Irrespective of whether the lamb was placed above or below the mother, initiating ventilation significantly reduced both UaBF and UvBF. While the reduction in UaBF was greater in lambs placed below the mother, so was the reduction in UvBF, again confirming the concept that flow into the placenta is a major determinant of flow out of the placenta. The greater reduction in $\mathrm{UaBF}$ in below lambs compared with above lambs was largely due to a marked reduction in end-diastolic UaBF, as there was no difference in peak systolic flows between groups. This indicates that gravity predominantly influences UaBF during diastole and is likely due to the arterial pressure head caused by placing the lamb below the mother.

During fetal life pulmonary vascular resistance (PVR) is high and PBF is low and the majority of right ventricular output flows through the ductus arteriosus (DA) into the dorsal aorta, rather than through the lungs, with much of it then flowing through the umbilical circulation. ${ }^{10}$ Lung aeration at birth decreases PVR and redirects right ventricular output so that $100 \%$ flows through the lungs, which markedly increases PBF. ${ }^{9} 16$ In addition, the decrease in PVR reverses the pressure gradient across the DA, which facilitates left to right flow across the DA, resulting in a large component of left ventricular output also contributing to PBF. ${ }^{10}$ In our study, the rapid increase in PBF associated with ventilation onset coincided with significant reductions in both UaBF and UvBF in both groups. Clearly, the reduction in UaBF (and as a result $\mathrm{UvBF}$ ) following ventilation onset, results from a redirection of both right and left (via left to right shunting through the DA) ventricular output through the pulmonary circulation, rather than through the placental circulation. This highlights the competitive interplay between the pulmonary and placental circulations for both left and right ventricular output during delayed UCC. The reduction in UaBF with ventilation onset was greatest in lambs placed below the midline and resulted in retrograde end-diastolic UaBF (indicated by negative values). This indicates that the pulmonary vascular bed eventually offers a lower resistance pathway for blood flow during diastole than flow through the placental vascular bed due to the vertical height difference. The finding that UvBF was also significantly reduced further confirms the interdependent nature of flows in these vessels. The redirection of left ventricular output into the pulmonary vascular bed rather than the placental vascular bed, via increased left to right flow across the DA, likely explains the higher PBF in lambs placed below the midline following ventilation onset. Indeed, we observed a strong negative correlation between end-diastolic UaBF and end-diastolic PBF, indicating that left to right shunting across the DA during diastole is largely responsible for the retrograde end-diastolic UaBF (figure $3 \mathrm{~F}$ ). Conversely, higher right to left shunting across the DA (indicated by negative PBF values during diastole) leads to higher enddiastolic UaBFs. 
These data clearly indicate that the changes in PVR and PBF associated with lung aeration have a much greater impact on the transitional circulation than gravity. This indicates that during delayed UCC, the infant's physiological state is the primary determinant of cardiovascular function and will likely determine the extent of placental to infant blood transfusion. A recent study in human infants has shown that, during DCC, UvBF is intermittent, markedly increasing during deep inspiratory efforts and ceasing or reversing during crying, ${ }^{5}$ presumably due to pressure changes within the abdomen and thorax. UaBF was also reduced and sometimes ceased during crying and at times flow became bi-directional, resulting in flow towards the placenta during systole and towards the infant during diastole. ${ }^{5}$ These effects of breathing/crying likely over-ride any circulatory changes that occur with changing body position alone. It is possible that placental to infant blood transfusion is increased during spontaneous breathing as a result of the subatmospheric intrathoracic pressures generated during inspiration. Indeed, deep inspiratory efforts are known to increase left to right shunting through the $\mathrm{DA}^{17}$ in human infants at birth, which also increase $\mathrm{UvBF}^{5}$ As such spontaneous breathing may be a major factor driving placental to infant blood transfusion and as our lambs where ventilated with positive pressure ventilation, this may explain why we were unable to detect any net increase in lamb blood volume.

\section{Effect of UCC}

UCC resulted in a further increase in PBF and systemic arterial pressure as has been described previously. ${ }^{3} 7$ While PBF was higher in below lambs, it was not significantly higher, and given the similarities between systemic oxygenation and pressure and cerebral oxygenation and blood flows between above and below lambs, there appears to be no physiological difference between the groups after UCC.

\section{Changes in blood volume (biotin)}

Using a biotin-labelled RBC technique, ${ }^{13}{ }^{14}$ we measured blood volume in the fetus + placenta and the blood volume remaining in the fetus following UCC. We were unable to detect any difference in blood volume remaining in the lamb irrespective of body position. We could not measure blood volume distribution between the fetus and placenta after the change in fetal position and prior to ventilation onset. As such, it is not known whether the absence of a difference is a consequence of ventilation having a greater impact than lamb position on blood volume, or that body position has a minimal impact on postnatal blood volume. However, given the vast majority of term infants breathe during delayed UCC, this finding likely represents the majority of infants undertaking delayed UCC, and are consistent with the recent clinical trial by Vain et al. ${ }^{6}$ Interestingly, Yao and Lind $^{2}$ also demonstrated no effect on placental transfusion when babies were placed either $10 \mathrm{~cm}$ above or below the introitus during uterine contractions. They considered that the lack of effect on placental transfusion was because the minimal change in hydrostatic pressure created would be lower than the uterine tone at relaxation. However, the uterine contractions themselves may have influenced their findings (discussed below in limitations section). The limitations on the length of umbilical cord in sheep prevented us from investigating whether placing the fetus lower or higher would influence the results. However, while it is possible that a greater vertical height may have produced a detectable blood volume transfer between lamb and placenta, this transfer would likely have been transient and relatively small.

\section{Cerebral oxygen kinetics}

Previous studies have shown that delayed UCC reduces intraventricular haemorrhage and improves motor outcomes in infants. $^{18} 19$ We have also shown that physiologically based UCC reduces cerebral hypoxia in preterm sheep, ${ }^{4}$ which is a major cause of perinatal brain injury in humans. ${ }^{20}{ }^{21}$ In this study, we found that changing body position had no effect on cerebral oxygenation, oxygen extraction or consumption. Importantly, ventilation prior to UCC, irrespective of body position, prevented any fall in cerebral oxygenation. This confirms that delayed UCC until after ventilation onset is effective at maintaining cerebral oxygenation.

\section{Limitations}

The ewes were anaesthetised throughout the experiment and were not in labour. Yao and Lind ${ }^{22}$ described rapid and stepwise increases in the redistribution of blood between infant and placenta coincident with uterine contractions during the third stage of labour. They postulated that uterine contractions increases blood transfer to the fetus, while blood flow to the placenta is favoured during uterine relaxation. While we could not investigate this phenomenon in this study, we are able to exclude the confounding influence of uterine contractions on the true effect of gravity. Further studies are required to determine the influence of maternal uterine contractions and the effects of spontaneous breathing versus positive pressure ventilation on placental blood flow during DCC at delivery.

\section{Summary}

Our study investigated the effect of gravity on UaBF and UvBF and on the cardiovascular transition at birth. Raising the fetus above the midline had no apparent influence on any variable, while placing the fetus below the midline caused small transient changes to $\mathrm{UaBF}$ and UvBF, which were similar in direction and magnitude, but no detectable changes in blood transfusion. Ventilation onset, however, caused significant, but similar effects on both $\mathrm{UaBF}$ and $\mathrm{UvBF}$ as well as on cardiovascular pressures and flows. We found that $\mathrm{UaBF}$ and UvBF are closely interrelated and that a reduction in flow in one vessel reduces flow in the other. Putting it simply, what comes out of the placenta is determined by what goes in, and therefore position change appears to not be an effective way of enhancing placental transfusion. Furthermore, the reduction in PVR following ventilation onset, redirected ventricular output away from UaBF and into the pulmonary circulation, causing a marked reduction in both $\mathrm{UaBF}$ and UvBF. Given that cerebral oxygenation was not different between groups at any stage of the experiment, and cardiovascular outcomes were similar after UCC, our study did not demonstrate any benefit of placing the fetus below the midline during delayed UCC, nor any adverse effect of placing the infant above the midline. This suggests that body position during delayed UCC does not influence the cardiovascular transition at birth. The limitations of the study prevent direct extrapolation to the human fetus during normal labour, but provide physiologic explanations to what occurs during the cardiovascular transition at birth.

Acknowledgements The authors would like to acknowledge the technical support of Katie Moores and Karyn Rodgers for their assistance with all experimental procedures described within this manuscript.

Contributors Conception or design of the work: SBH, KJC, ABtP, EMW, AWG, MK and GRP. Acquisition, analysis or interpretation of data for the work: SBH, VVZ, KJC, JVV, AM, AWG, MK, ABtP, EMW and GRP. Drafting the work or revising it critically for important intellectual content: SBH, VVZ, KJC, JVV, AM, AWG, MK, ABtP, EMW and GRP. 
Funding This research was supported by National Institutes of Health R01HD072848-01A1, National Health and Medical Research Council (NH\&MRC) Program Grant (606789), NH\&MRC Project Grant (1067615) NH\&MRC Research Fellowships (GRP: 1105526 and SBH: 545921) and the Victorian Government's Operational Infrastructure Support Program.

Competing interests None declared.

Provenance and peer review Not commissioned; externally peer reviewed.

Open Access This is an Open Access article distributed in accordance with the Creative Commons Attribution Non Commercial (CC BY-NC 4.0) license, which permits others to distribute, remix, adapt, build upon this work non-commercially, and license their derivative works on different terms, provided the original work is properly cited and the use is non-commercial. See: http://creativecommons.org/ licenses/by-nc/4.0/

\section{REFERENCES}

1 Wyllie J, Perlman JM, Kattwinkel J, et al. Part 7: Neonatal resuscitation: 2015 International Consensus on Cardiopulmonary Resuscitation and Emergency Cardiovascular Care Science with Treatment Recommendations. Resuscitation 2015;95:e169-201.

2 Yao AC, Lind J. Effect of gravity on placental transfusion. Lancet 1969;2:505-8.

3 Bhatt $\mathrm{S}$, Alison BJ, Wallace EM, et al. Delaying cord clamping until ventilation onset improves cardiovascular function at birth in preterm lambs. J Physiol 2013;591: 2113-26.

4 Polglase GR, Dawson JA, Kluckow M, et al. Ventilation onset prior to umbilical cord clamping (physiological-based cord clamping) improves systemic and cerebral oxygenation in preterm lambs. PLoS ONE 2015;10:e0117504.

5 Boere I, Roest AA, Wallace $E$, et al. Umbilical blood flow patterns directly after birth before delayed cord clamping. Arch Dis Child Fetal Neonatal Ed 2015;100:F121-5.

6 Vain NE, Satragno DS, Gorenstein AN, et al. Effect of gravity on volume of placental transfusion: a multicentre, randomised, non-inferiority trial. Lancet 2014;384:235-40.

7 Rudolph AM. Fetal and neonatal pulmonary circulation. Annu Rev Physiol 1979;41:383-95.

8 Dawes GS. Foetal and neonatal physiology: a comparative study of the changes at birth. Chicago: Year Book Medical Publishers, Inc., 1968.
9 Rudolph AM. Fetal and neonatal pulmonary circulation. Am Rev Respir Dis 1977:115(Pt 2):11-18

10 Crossley KJ, Allison BJ, Polglase GR, et al. Dynamic changes in the direction of blood flow through the ductus arteriosus at birth. J Physiol 2009;587(Pt 19): 4695-704.

11 Polglase GR, Wallace MJ, Morgan DL, et al. Increases in lung expansion alter pulmonary hemodynamics in fetal sheep. J Appl Physiol 2006;101:273-82.

12 Polglase GR, Moss TJ, Nitsos I, et al. Differential effect of recruitment maneuvers on pulmonary blood flow and oxygenation during HFOV in preterm lambs. J Appl Physiol 2008;105:603-10.

13 Mock DM, Lankford GL, Widness JA, et al. Measurement of red cell survival using biotin-labeled red cells: validation against $51 \mathrm{Cr}$-labeled red cells. Transfusion 1999:39:156-62.

14 Mock DM, Matthews NI, Strauss RG, et al. Red blood cell volume can be independently determined in vitro using sheep and human red blood cells labeled at different densities of biotin. Transfusion 2009;49:1178-85.

15 Balegar KK, Stark MJ, Briggs N, et al. Early cerebral oxygen extraction and the risk of death or sonographic brain injury in very preterm infants. J Pediatr 2014;164:475-80.e1.

16 Lang JA, Pearson JT, Binder-Heschl C, et al. Increase in Pulmonary blood flow at birth: role of oxygen and lung aeration. J Physiol 2016;594:1389-98.

17 van Vonderen JJ, Roest AA, Walther FJ, et al. The influence of crying on the ductus arteriosus shunt and left ventricular output at birth. Neonatology 2015;107: 108-12.

18 Mercer JS, Vohr BR, Erickson-Owens DA, et al. Seven-month developmental outcomes of very low birth weight infants enrolled in a randomized controlled trial of delayed versus immediate cord clamping. J Perinatol 2010;30:11-16.

19 Mercer JS, Vohr BR, McGrath MM, et al. Delayed cord clamping in very preterm infants reduces the incidence of intraventricular hemorrhage and late-onset sepsis: a randomized, controlled trial. Pediatrics 2006;117:1235-42.

20 Khwaja 0, Volpe JJ. Pathogenesis of cerebral white matter injury of prematurity. Arch Dis Child Fetal Neonatal Ed 2008;93:F153-61.

21 Volpe JJ. Brain injury in premature infants: a complex amalgam of destructive and developmental disturbances. Lancet Neurol. 2009;8:110-24.

22 Yao $A C$, Lind J. Blood flow in the umbilical vessels during the third stage of labor. Biol Neonate 1974;25:186-93. 